\title{
Interprofessional Collaboration Experiences of Student Physical Therapists and their Clinical Instructors in Orthopedic versus Neurologic Rehabilitation Settings
}

\author{
Rylie Kohls, Sascha Smith, Heather Braden* and Teresa Huckaby \\ Department of Physical Therapy, Angelo State University, USA
}

*Corresponding author: Heather Braden, Department of Physical Therapy, Angelo State University, USA

To Cite This Article: Rylie Kohls, Sascha Smith, Heather Braden, Teresa Huckaby. Interprofessional Collaboration Experiences of Student Physical Therapists and their Clinical Instructors in Orthopedic versus Neurologic Rehabilitation Settings. 2020 - 8(6). AJBSR.MS.ID.001339. DOI: 10.34297/AJBSR.2020.08.001339.

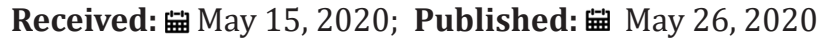

\begin{abstract}
Objective: To assess the differences in how student physical therapists (SPTs) and their clinical instructors (CIs) utilize and recognize interprofessional collaboration (IPC) in the neurologic and orthopedic physical therapy settings.

Design: This study is a mixed methods, survey research study. In order to gather data, two surveys using Likert scales and free response questions were administered. An independent samples t-test was utilized to interpret differences in CI and SPT scores in the same setting, as well as comparing CI scores to those from the other setting. A paired t-test was used to identify differences in doctor of physical therapy (DPT) student IPC experiences in the orthopedic and neurologic settings.
\end{abstract}

Setting: The data collection for this study took place on a 10-week orthopedic clinical practicum and in the first 10 weeks of a 12 -week neurologic clinical practicum.

Participants: N=26 DPT students consented to participate in this study. Participant mean age was $26.92+3.0$ years, with 16 males. There were 19 SPTs who completed the study in the orthopedic setting, and 16 DPT students in the neurologic setting. A total of 17 CIs in the orthopedic and 13 CIs in the neurologic setting participated.

Main Outcome Measure: Performance of IPC via the Interprofessional Collaborator Assessment Rubric (ICAR) by students, and perception of SPT and CI self-IPC via the Modified Index of Inter professional Collaboration (MIIC) were utilized in this study.

Results: There were statistically significant differences found between groups and settings when comparing IPC utilization.

Conclusion: IPC experiences varied between SPTs and CIs, as well as between the orthopedic and neurologic settings. This study found that IPC is utilized more in the neurologic setting and CIs report using IPC more than SPTs in the same setting. CIs report their students not taking advantage of all IPC opportunities available, while SPTs report using IPC more than CIs think they are utilizing it in each setting.

Keywords: Interprofessional collaboration; Physical therapy; Interprofessional education

\section{Introduction}

Interprofessional collaboration (IPC) within the healthcare system is crucialin order to providethemostbeneficial,cost-effective, and highest quality forms of treatment. In 2009, the American Physical Therapy Association (APTA) reframed professional goals towards a patient relationship paradigm where "PTs are effective and thrive as part of a collaborative, interprofessional health care team with patients and families at its focus" [1]. IPC is defined by a diverse team of health care professionals working cohesively to improve lives via the quality care of patients, families, and their communities, and as such, becomes even more important when treating patients with a variety of medical needs [2]. For those patients who require a multitude of health care professionals to be part of their medical team, it is imperative that professionals communicate, plan, and execute a treatment plan that is beneficial to the patient both medically and financially, as their needs cannot be met by one discipline alone [3]. 
By working together, health care professionals are able to give the best care at the fairest cost without over-utilizing the healthcare system. This is not always something that comes naturally, as many health care professionals need to take the time to learn what other fields can offer and how they can work cohesively to increase efficiency and quality of care. Possible barriers to the practice of IPC include an absence of interprofessional education (IPE), both academically and clinically, as well as a lack of individual motivation for IPE experiences and subsequent IPC. One study found that IPC, which fulfilled the student's needs for competence, relatedness, and autonomy, increased motivation for the use of IPC in the future [4]. Healthcare professionals who are successfully practicing IPC are therefore presenting patients with an image of a united group of experts doing all they can to help. Studies have shown that interprofessional education (IPE) regarding IPC is a way to address "health care issues such as efficiency and patient safety", while also improving patient outcomes, communication skills, and job satisfaction of both SPTs and practicing physical therapists [5].

Recently, the literature has focused on IPC and the steps that are deemed necessary to successfully work together to treat patients. The literature includes patients who are being treated by multiple health fields due to the complexity of their conditions. These steps encompass patient identification and root cause analysis, engagement of the clinical partnership, shared infrastructure, facilitating effective team culture, ongoing management, and joint celebration of success [6]. Patient identification and root cause analysis includes education of the patient's team, and collecting information on the cause of the diagnosis to better create and implement a treatment plan. Clinical partnership involves removing competition between healthcare providers and providing patient-centered care for their individually specific needs and desires. Within this step, each professional is putting forth their own expertise. Shared infrastructure involves the creation of documentation and other elements that encourages ongoing IPC, such as the Business Associate Agreement (BAA). Facilitating an effective team culture is critical for IPC because mutual respect and recognition of individual strengths and limitations between team members is necessary for the best possible patient treatment. The final two steps are ongoing management and celebration of success, which involve forming professional relationships with other facilities in the community, and sharing successes and reinforcing relationships between organizations [6].

One study has suggested that to implement IPC effectively as a healthcare professional, there needs to be an emphasis on IPE in the academic setting as well as clinical IPE that is guided by the clinical instructor (CI) [5]. In the United States, IPE is becoming more of a point of focus within physical therapy programs. The Commission of Accreditation of Physical Therapy Education (CAPTE) mandated accredited PT programs "include both didactic and clinical IPE" learning opportunities beginning in 2018, and specific standards are being created [1]. Focuses on IPE and IPC early in DPT curriculums are imperative when considering the impact it has with future employers, whose goals are "improving patient outcomes" through the use of collaborative practice strategies [7].

Depending on the program, there are IPE courses given in the academic setting, while other programs expect most, if not all, IPE to occur within the clinical setting under the supervision of the CI. When interviewed, many CI's stated that the university's expectations of clinical IPE were unclear, as IPE was not clearly delineated in provided clinical performance tools for student evaluations [5]. The use of specifically focused IPE courses may also be of benefit, as one study involving medical and DPT students found that when working together to complete a neurologic examination after an IPE course, the result was more comfort when treating individuals with disabilities [3] It has also been found that after a single IPE session, health care professional students reported increased knowledge of their peers' professions as well an increased desire to work in an interprofessional setting, showcasing the impact of experiences involving this topic.8 Within the acute care setting, clinical IPE tends to be informally approached according to the $\mathrm{CI}$, making it inconsistent between programs, settings, and specific situations [5]. This lack of consistency demonstrates a need for more research to be conducted regarding IPE in clinical settings.

Within inpatient facilities, such as the neurologic rehabilitation settings of this study, there are more opportunities for IPC to occur due to the proximity of multiple health care providers. While this does have many benefits, the differences among health care specialties can cause barriers to IPC that must be overcome. It has been found that communication inconsistencies are the cause of up to two-thirds of sentinel evens, proving that IPC is a skill set of grave importance in all settings, but especially when treating complex patients in inpatient settings [9]. This study, and those like it, are crucial in highlighting the need for IPC and IPE in these settings.

Within the outpatient setting, there are limited direct opportunities for physical therapists to practice IPC due to the vastly reduced variety of other medical professionals available in the facility. In light of this, the outpatient orthopedic setting is one of the focus points of this study. In recent literature, researchers found that most interprofessional work occurs in the inpatient setting with very little demonstrated in outpatient. With a large majority of PTs working in outpatient settings, there is much room for improvement [1]. The previously mentioned researchers also stated that students have less opportunities for IPE in outpatient settings as compared to inpatient, posing the question if students will need more IPE in academic settings [1]. The authors of this current study hypothesize that IPC will be utilized more within the neurological inpatient settings by CIs and SPTs, and that due to limited clinical experiences, students will perceive less IPC in the same settings than their CIs. 


\section{Methods}

\section{Subjects}

The participants of this study were recruited from the Doctor of Physical Therapy (DPT) program Class of 2019 at Angelo State University. Consenting students ( $\mathrm{N}=26$ SPTs, mean age $26.92 \pm 3.0$ y, 16 males, 10 females) who were enrolled in and completed both the 10 -week.

Musculoskeletal Practicum and the 12-week Neuromuscular Practicum, as well as the clinical instructors (CIs) who supervised each student in both settings, were recruited to be subjects for this study. A total of 17 CIs from the outpatient orthopedic rehabilitation setting consented to participate in this study. From the neurologic rehabilitation setting, a total of 13 CIs consented to participation. In all settings, students practiced 40 hours per week under the supervision of their CI. Prior to initiating the study, Institutional Review Board approval was granted and each of the participants provided consent prior to participation in the study's methods.

\section{Testing Procedure}

All participants of this study were enrolled in the required courses, completed the required hours, and met expectations in each of the two specified settings. Students and CIs both consented to completing the provided surveys during the $10^{\text {th }}$ week of each rotation regarding their IPC experience. Students were assessed by the CIs utilizing the ICAR. All of which was completed during the tenth week of both the orthopedic and neurologic rotations. A confidential program was utilized for data collection, and each participant was assigned a number in order to maintain both confidentiality and anonymity. Only the data analyst had access to the master code, and interpreted the information before providing the researchers with the anonymous results.

\section{Survey}

This study utilized a mixed methods survey research design. Surveys utilized in this study were the Modified Index of Interprofessional Collaboration (MIIC) and the Interprofessional Collaborator Assessment Rubric (ICAR), both consisting of Likert scales and short free response sections. The ICAR was only completed by the CIs, and contained questions that addressed and assessed how well the student performed IPC throughout their practicum. The ICAR included a Likert scale with four options ranging from "does not do" or minimal demonstration of qualities, to "consistently does" or mastery of qualities, when assessing the regularity of interprofessional collaboration. The ICAR also included six comment sections that were summarized into themes consistent across all gathered replies. The themes addressed in this survey are communication, collaboration, roles and responsibility, collaborative patient/client-family centered approach, team functioning, and conflict management/resolution. The MIIC survey's verbiage was minimally modified to more accurately address the population of this study, with permission from the original creators, and was completed by both CIs and students. The primary content of the MIIC was in original form. This survey asked questions to assess the opinions and views of IPC in their particular setting, as well as how regularly IPC is utilized by both the participant and other healthcare professionals they work with. The MIIC includes a Likert scale with choices ranging numerically from one to seven with one stating "strongly disagree" and seven stating "strongly agree". Any results above 5, labelled as "agree", showed significant knowledge and use of IPC. The MIIC also included two free response question opportunities. Please see Appendix $1 \& 2$ for images of both scales.

\section{Statistical Analysis}

The registered SNAP Survey Software was utilized to collect and store the obtained data from both the students and their CIs in each of the two settings. This software program encrypts all information being entered and received from the site, and has fully secure connections. Each student and CI were assigned a specific code by the aforementioned software in order to make all information anonymous, and categorize gathered data accordingly and appropriately for those who complete the orthopedic and neurologic clinical practicum's. The information obtained from the surveys were stored in a secure database and analyzed by the Statistical Package for the Social Sciences (SPSS) 18.0 software (Chicago, IL., USA).

In order to compare the orthopedic and neurologic rehabilitation settings for both the students and CIs, an independent samples t-tests was used for the CIs across settings, while a paired t-test for the same students across the two settings. The t-tests were used to compare two ratio average of scores. Statistical differences between the students and the CIs in the same setting were determined by utilizing a between group design with an independent t-test.

Statistically significant results were based on a p-value of $\leq 0.05$. In order to determine equality of variance, Levene's test was used. An independent t-test was used for the IPC components both individually and as an overall mean. The independent factors that were considered were the gender of the student, CI age, CI gender, and the number of years the $\mathrm{CI}$ has worked in that setting. The dependent variable was determined by a regression analysis that identified the best predictor of change in survey results between the two settings being addressed. Standard Error for the Measurement (SEM) was used to calculate the reliability, as well as to determine the minimal detectable change in order to assess the minimal change not due to a measurement error. SEM is related to test reliability and $=$ standard deviation $* \sqrt{ }$ (1-the reliability coefficient).

Reliability coefficient of 0.90 is assumed, as reported by Mangione et al., to determine the standard error of measurement.10 
The minimal detectable change was calculated $($ MDC90 $=1.64 \times$ $\mathrm{SEM} \times \sqrt{2}$ ) at the $90 \%$ confidence level.

\section{Results}

By viewing histograms, skewness kurtosis, and Shapiro Wilk tests, it was determined that the data was normally distributed. Levene's test for equality of variance was used to demonstrate equal variances across groups.

Statistical analysis via independent samples t-tests demonstrates statistically significant differences between the orthopedic and neurologic settings when comparing the amount of IPC. The CI IPC scores for the neurologic setting (mean=6.34, $\mathrm{SD}=.67, \mathrm{~N}=13$ ) were significantly higher than those in the orthopedic setting (mean=5.88, $\mathrm{SD}=.61, \mathrm{~N}=17$ ), $\mathrm{t}=36.5, \mathrm{df}=17, \mathrm{p}=.004$; with a Cohen's d of 0.34 which indicates a small effect size. The student's IPC in each setting was also evaluated with a paired samples t-test. The neurologic setting (mean=6.10, $\mathrm{SD}=.86, \mathrm{~N}=19$ ) was again, significantly higher than that of the students in the orthopedic setting (mean=5.46, $\mathrm{SD}=1.21, \mathrm{~N}=16$ ), $\mathrm{t}=23.66, \mathrm{df}=15, \mathrm{p}=.006$; with a Cohen's d of 0.58 which indicates a medium effect size (Figure 1).

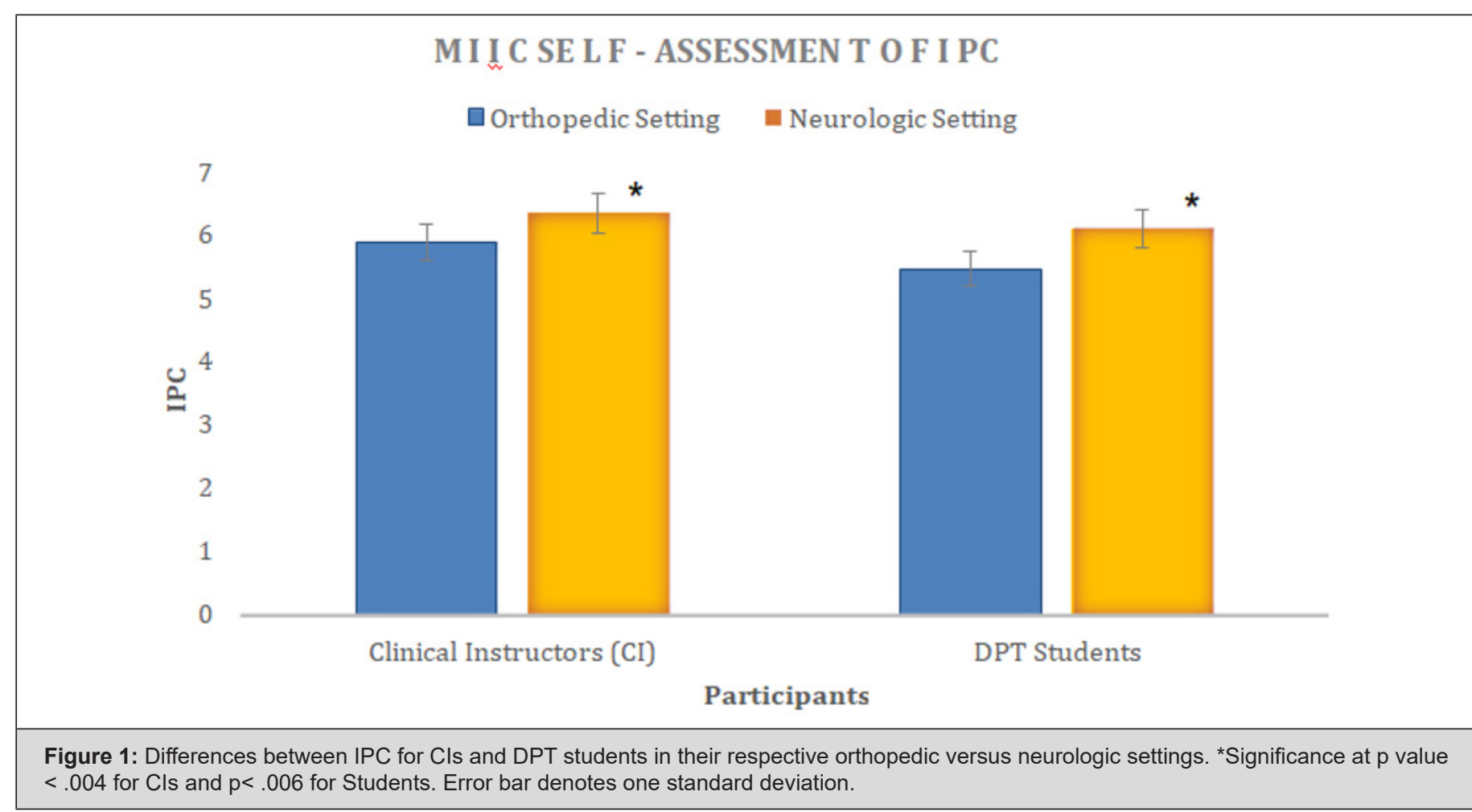

$<.004$ for Cls and $p<.006$ for Students. Error bar denotes one standard deviation.

\section{ICAR \\ PERFORMANCE- BASED IPC}

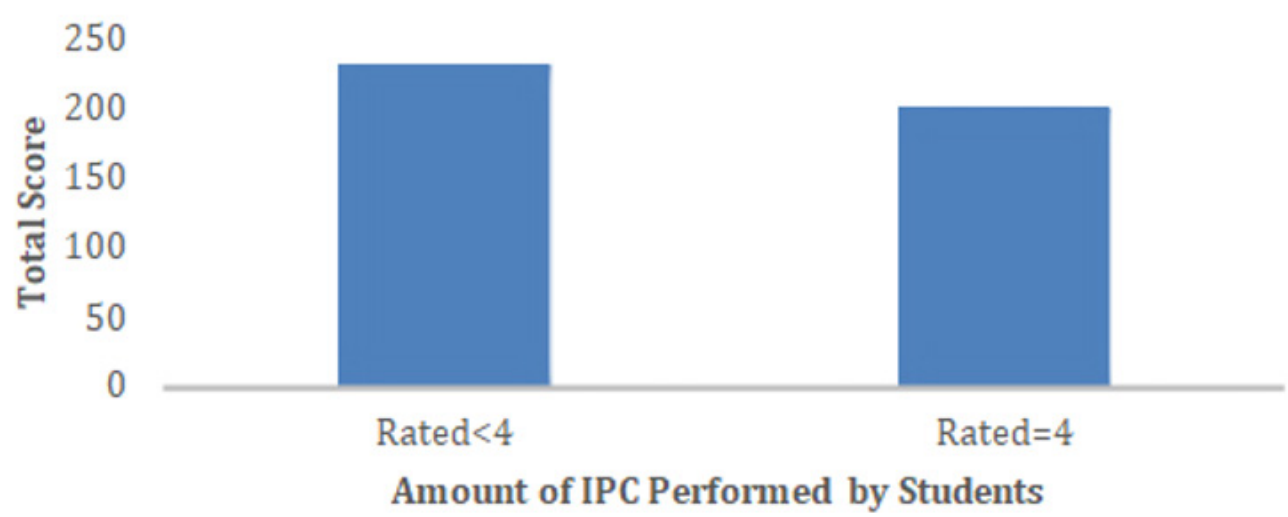

Figure 2: Demonstrates performance-based student IPC in both orthopedic and neurologic settings. $4=$ high IPC demonstrated while $<4=$ less IPC by the student. 
When considering DPT student IPC performance across both settings, 200 total scores on the ICAR were ratings of 4, indicating high levels of IPC. On the other hand, 231 total scores on the ICAR indicated the students lacked high levels of IPC utilization in the clinical environment as seen by the clinical instructor (Figure 2). There were three statistically significant odds ratios demonstrated by the Vassar College Odds Ratio Calculator. CIs were 1.7 times more likely to mark 6 ("Agree") or 7 ("Strongly Agree") on the MIIC than the SPTs, when asked about the amount of IPC in their setting
(95\% CI 1.33-2.26). CIs were also 3.2 times more likely to score 6 or 7 on the MIIC regarding their own high levels of IPC in their setting compared to the ratings they gave the students on the performancebased ICAR in that same setting (95\% CI 2.45-4.23). Finally, the SPTs rated themselves at a 6 or 7 on the MIIC, for the amount of IPC they experienced in their setting, 1.9 times more often than their CIs gave them a 4 (rating consistent use of IPC performance) on the performance-based ICAR (95\% CI 1.43-2.41), ( $p=<.0001$ for all of the above listed odds ratios) (Figure 3).

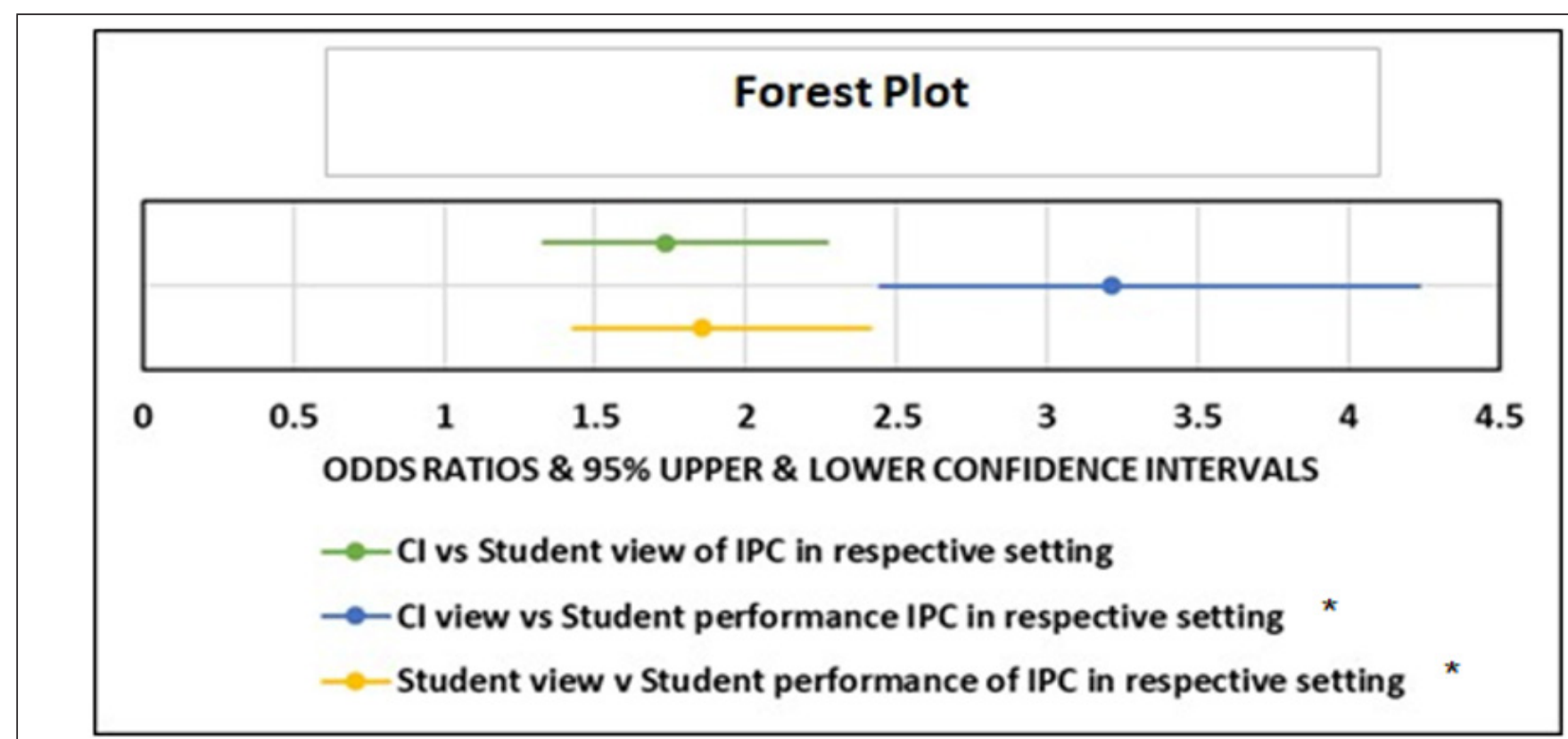

Figure 3: Demonstrates three statistically significant odds ratios regarding IPC for Clinical Instructors $(\mathrm{Cl})$ and the DPT students. *Significance at $p$ value $<.0001$ with $95 \% \mathrm{Cl}$.

\section{Discussion}

This study's aim was to compare interprofessional collaboration as seen from the perspective of SPTs in contrast to that of their CIs during an orthopedic and neurologic clinical practicum. With this knowledge, it is possible to open the discussion about the need for increased IPE within the academic curriculum to supplement and prepare the students for what they receive in their clinical practicums. This is an important focus both academically and clinically due to the increasing need of IPC throughout the healthcare setting, due to the current lack of consistency in delivery of necessary IPE.

When the participants of this study were asked their opinions about IPC in a free response section of the MIIC, one student reported that it allowed for "open communication, quick referrals, and desire to supply the best care possible to all patients." This was reflected in the other students' comments made throughout the surveys as well. There was agreement between SPTs and CIs that, when IPC is utilized properly, it benefits their patients.

\section{Principal Findings}

As discussed above, this study found IPC to be utilized more commonly by both practicing clinicians and SPTs in the neurologic settings when compared to orthopedic settings. This is understandable due to the complexity of the neurologic patient's treatment, requiring a complete health care team to appropriately address their diagnoses and comorbidities. In this population, it is easy to see how efficient and respectful IPC can directly lead to increased patient care, decreased patient stay, and improved overall satisfaction by the patient and family. It is also understandable that there is a significant difference between neurologic and outpatient settings due to the availability of other professions in the neurologic settings. PTs in outpatient settings are required to actively seek out the input from other professions, so it is left to them to be an active participant in IPC in the orthopedic, outpatient setting. This is yet another reason it is so crucial to encourage therapists and students to seek out IPC in every setting.

In order to have the comprehensive IPC that is most beneficial to patients, IPE is crucial during the provider's education. IPC may not 
always come naturally for each physical therapist, especially entry level therapists, so addressing the topic early and often throughout their education is essential. Studies have shown that introducing IPE early can increase students' attitudes and perception of the other health care providers they work alongside [8]. Not only do all professions need to respect their counterparts, but it is of the utmost importance that they understand their roles, responsibilities and scope of practice, as well as how to communicate with those who come from different backgrounds [8,11]. Kim et al. discusses in their article a significant number of studies that demonstrate, after students receive just one session of IPE, their attitudes toward IPE increased, along with their perceptions and understanding of other professions [8].

This study also found that SPTs were more likely to rate themselves as practicing more IPC in their clinical settings than their CIs reported the students practiced. Other studies have reported "generally favorable perceptions of readiness for interprofessional learning" are seen by both incoming and current physical therapy students [12]. Students are generally open to learning about interprofessional collaboration, and in being somay place higher value on the IPC they are participating in than their CIs, that see it regularly. Student reports shown in one study state that IPE given for formal credit, with a larger percentage of cases and less presentations, would be received favorably.12 This could indicate the need for more consistency in IPE for students both from their CIs in clinic and from their professors academically. There are many different IPC courses that are currently being studied, which could provide a foundation for future studies to formulate a baseline IPE requirement $[4,8,12-14]$. As in the current study and the others demonstrated above, increased IPE in the academic and clinical settings would prove favorable by the students.

Finally, this study also found that CIs perceived they participated in more IPC than their students in each setting. They reported feeling as though their students needed more IPC practice, and that students did not take full advantage of all the opportunities available. One study found, when looking at IPE courses that included many different medical professionals, that the SPT's professional perspectives were fairly unknown to both nursing and medical school students [4]. This caused the SPTs to not take part in IPC to the full extent their role required. They found that, after a course with all the professions involved, SPTs were more apt to involve themselves in the discussion after their role had been defined [4]. This finding demonstrates the need of IPE across the healthcare professional spectrum. In order to provide the best quality care to patients, each professional in the health care team must contribute in their area of expertise.

The results of this study demonstrate the passive nature of SPTs to take full advantage of IPC opportunities as seen by their CIs. This finding was discussed likewise in a study completed by Visser et. al.
These findings give credence to the benefit of multidisciplinary IPE both in academic curricula, as well as continuing education courses.

The awareness of the need for IPC has increased, so it is necessary that physical therapy schools' curriculum should reflect this need [7]. Not only does IPE in academics increase the ability of students to participate more fluently in IPC during their clinical rotation, it is also an area considered by future employers. In an article by the APTA, the importance of IPE experiences in potential employees was discussed. APTA states that IPE on a resume gives the employer the knowledge that the graduate PT will be flexible, innovative, adaptable, and open to change [7]. These are qualities employers look for in new hires. So, including IPE in academics, in addition to what the students are receiving clinically, is just another step institutions can take to better prepare their students to be quality physical therapists sought out by employers [7].

\section{Limitations}

The primary limitation of this particular study is small sample size. With only one class of SPTs', as well as the number of incomplete surveys, this study could benefit from an increased number of participants. There is also a bias present due to SPTs attendance to the same academic institution and the majority of clinical practicums being located in the same state. More variety would be a valuable addition to this study as far as clinical locations and academic institutions.

\section{Future Studies}

Future studies could benefit from a larger sample size, as well as a more varied population as discussed above. Future studies could also benefit from adding a source of IPE in the school to compare to in clinic sources as viewed by the SPTs. This would give a better view of how IPE preparation prior to clinical practice benefits SPT's during their clinical practicums.

\section{Conclusion}

As hypothesized, there were statistically significantly differences in the utilization of IPC in the orthopedic and neurologic settings by both SPTs and their CIs, with the neurological setting having greater IPC. In each setting, CIs believed there were opportunities for IPC, and that they, as clinicians, utilize these opportunities frequently and appropriately in the care of their patients. This is encouraging as it showcases the importance the physical therapists place on IPC and the opportunities the SPTs have while on clinical practicums. Lastly, the SPTs in this study reported practicing more IPC than their CI believed they did over their practicum. This difference could be due to a number of reasons, but illustrates the need for further SPT self- evaluation and SPT/CI communication on the subject. The addition of thorough IPE courses in the academic setting, as well as in the clinical settings, would greatly benefit not only SPTs, but students in all health care professions to relay their strengths and responsibilities in patient care. The overall goal of IPC is to improve 
medical care and outcomes of each patient. That starts with the learning of communication techniques and teamwork between all health care providers involved.

\section{References}

1. Fitzsimmons A, Topp K, O’Brien B (2017) Investigation into Physical Therapist Students' Interprofessional Experiences During an 8-Week Clinical Clerkship: A Qualitative Study. Journal of Physical Therapy Education 31(2): 44-53.

2. Toh L, Lai P, Othman S, Wong K, Low B, et al. (2017) An analysis of interprofessional collaboration in osteoporosis screening at a primary care level using the D'Amour model. Research In Social And Administrative Pharmacy 13: 1142-1150.

3. Garavatti E, Tucker J, Pabian P (2018) Utilization of an interprofessional integrated clinical education experience to improve medical and physical therapy student comfort in treating patients with disabilities. Education for Health: Change in Learning \& Practice (3): 155-162.

4. Visser CLF, Kusurkar RA, Croiset G, Ten Cate O, Westerveld HE (2018) Students' motivation for interprofessional collaboration after their experience on an IPE ward: A qualitative analysis framed by selfdetermination theory. Medical Teacher 41(1): 44-52.

5. Chau J, Denomme J, Cott C (2011) Inter-professional education in the acute-care setting: the clinical instructor's point of view. Physiotherapy Canada 63(1): 65-75.

6. Hardin L, Kilian A, Spykerman K (2017) Competing health care systems and complex patients: An inter-professional collaboration to improve outcomes and reduce healthcare costs. Journal of Inter professional Education \& Practice 7: 5-10.
7. Smith LJ, Curtis CP, Yorke A (2018) Student considerations when selecting an entry-level physical therapy program. Does interprofessional education curriculum matter? Journal of Interprofessional Education \& Practice 11: 1-6.

8. Kim YJ, Radloff JC, Stokes CK, Lysaght CR (2019) Interprofessional education for health science students' attitudes and readiness to work interprofessionally: a prospective cohort study. Brazilian Journal of Physical Therapy / Revista Brasileira de Fisioterapia 23(4): 337-345.

9. Weaver A, Callaghan M, Cooper A, Brandman J, O'Leary K (2015) Original Contribution. Assessing Interprofessional Teamwork in Inpatient Medical Oncology Units. Journal of Oncology Practice 11(1): 19-22.

10. Mangione KK, Craik RL, McCormick AA, Heather L, Meaghan B, et al. (2010) Detectable changes in physical performance measures in elderly African Americans. Physical Therapy 90(6): 921-927.

11. Byrne Á, Pettigrew CM (2010) Knowledge and attitudes of allied health professional students regarding the stroke rehabilitation team and the role of the Speech and Language Therapist. International Journal of Language \& Communication Disorders 45(4): 510-521.

12. Zeeni N, Zeenny R, Hasbini-Danawi T, Nadia A, Maya B, et al. (2016) Student perceptions towards interprofessional education: Findings from a longitudinal study based in a Middle Eastern university. Journal of Interprofessional Care 30(2): 165-174.

13. Wojciechowski M (2013) The Benefits of Interprofessional Collaborative in Clinical Education. PT in Motion.

14. Avrech Bar M, Katz Leurer M, Warshawski S, Itzhaki M (2018) The role of personal resilience and personality traits of healthcare students on their attitudes towards inter professional collaboration. Nurse Education Today 61: 36-42. 